\title{
Optical quantum memory for ultrafast photons using molecular alignment
}

\author{
G.S. Thekkadath, K. Heshami, D.G. England, P.J. Bustard, B.J. Sussman, and M. Spanner* \\ National Research Council of Canada, 100 Sussex Drive, Ottawa, Ontario, K1A 0R6, Canada
}

\begin{abstract}
The absorption of broadband photons in atomic ensembles requires either an effective broadening of the atomic transition linewidth, or an off-resonance Raman interaction. Here we propose a scheme for a quantum memory capable of storing and retrieving ultrafast photons in an ensemble of twolevel atoms by using a propagation medium with a time-dependent refractive index generated from aligning an ensemble of gas-phase diatomic molecules. The refractive index dynamics generates an effective longitudinal inhomogeneous broadening of the two-level transition. We numerically demonstrate this scheme for storage and retrieval of a weak pulse as short as $50 \mathrm{fs}$, with a storage time of up to $20 \mathrm{ps}$. With additional optical control of the molecular alignment dynamics, the storage time can be extended about one nanosecond leading to time-bandwidth products of order $10^{4}$. This scheme could in principle be achieved using either a hollow-core fiber or a high-pressure gas cell, in a gaseous host medium comprised of diatomic molecules and a two-level atomic vapor at room temperature.
\end{abstract}

\section{INTRODUCTION}

Controlling light-matter interfaces is essential to further develop optical quantum information processing elements. Optical quantum memories are one of the primary goals of this development, and their applications [1, 2] are likely to extend beyond their role in quantum repeaters [3]. Many recent efforts have been devoted to their implementation in atomic ensembles [4], and in particular, memories compatible with ultrafast light are desirable for their large time-bandwidth products and fast operational speeds [5-7].

Absorbing broadband photons in atomic ensembles requires either a controlled inhomogeneous broadening of the atomic transition or an off-resonant Raman interaction, such as Raman memories [8], atomic frequency combs [9], and gradient echo memories (GEMs) [10]. This latter approach uses a longitudinal controlled reversible inhomogeneous broadening of the transition frequency of the medium to control the photon storage and retrieval [11]. The inhomogeneous broadening is induced by a spatial gradient in an external static field and modifies the transition frequency of the atoms. If the broadening is reversed, the time-reversal symmetry of the Maxwell-Bloch equations predicts that the absorbed light is subsequently re-emitted 12]. GEMs have been realized with a spatially-dependent linear Stark or Zeeman shift in rare-earth-doped crystals and warm atomic vapors [10, 13 15]. The strength and reversal timescale of the external field impose technical and fundamental limitations on the operational bandwidth of the GEM, and consequently the experimental demonstrations have remained limited to microsecond-duration pulses.

Ref. [16] showed that having a propagation medium with a linear time-dependent refractive index is equivalent to the longitudinal (spatial) inhomogeneous broadening in a GEM. The time-dependent refractive index can be generated in a host crystal exposed to a varying electric field, and induces a linear spatially-dependent frequency shift in the propagating signal pulse that mimics the effect of a varying resonance atomic frequency. This scheme relies on the temporal modulation of an external electric field which cannot be switched quickly enough to store ultrafast photons.

In this paper, we employ molecular alignment techniques to engineer the ultrafast refractive index dynamics necessary for storing femtosecond pulses in an atomic ensemble. Molecular alignment is a well-studied phenomenon [17] where a non-resonant ultrafast laser pulse impulsively generates a coherent excitation in the rotational levels of an ensemble of molecules via the dynamic Stark effect [18]. It has been used to shape light pulses [19 21], dissociate diatomic molecules [22], in optical imaging [23], and for quantum control scenarios [2426]. We propose to use such an interaction in a gaseous host medium containing diatomic molecules and twolevel atoms (e.g. warm atomic vapor) to optically prepare refractive index dynamics which can be used for a GEM-type storage and retrieval of ultrafast photons. As a proof-of-principle, we numerically simulate this interaction for the case of a mixed $\mathrm{CO}_{2} /{ }^{87} \mathrm{Rb}$ gas, and store the signal using the $\mathrm{D}_{1}$ transition $(795 \mathrm{~nm})$ of ${ }^{87} \mathrm{Rb}$ atoms [27].

The paper is organized as follows: in Sec. III, we briefly explain the proposed scheme, and present the necessary molecular alignment and Maxwell-Bloch equations to numerically simulate the process of storing and retrieving a signal pulse. In Sec. III, we discuss the results of the simulation and propose a possible experimental implementation. Finally in Sec. IV] we conclude the paper.

\section{THEORY}

\section{A. General Concept}

The proposed scheme is as follows (see Fig. 11): (i) ( ensemble of diatomic gas molecules and generates a time-dependent refractive index $n(t)$ in the propagation 


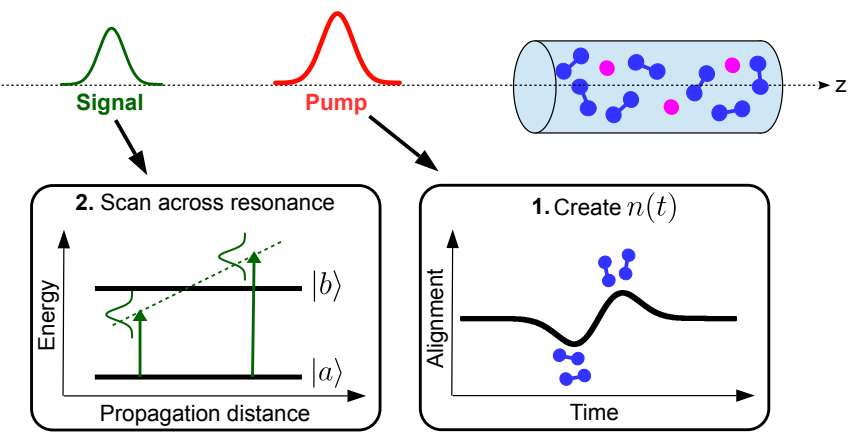

FIG. 1. Input pulse sequence for the memory scheme. A pump pulse and a delayed signal pulse are sent into a mixed gas medium comprised of $\mathrm{CO}_{2}$ molecules (blue) and ${ }^{87} \mathrm{Rb}$ atoms (pink). Step 1: The pump generates time-dependent molecular alignment, which causes a corresponding timedependent refractive index $n(t)$ in the medium. Step 2: The delayed signal pulse experiences the $n(t)$ as it propagates through the medium. For specific delays, the $n(t)$ causes the bandwidth of the signal to be scanned across the atomic resonance and thus absorbed in the two-level atoms. Step 3: (not shown) At a later time, the $n(t)$ is reversed due to the natural rotational dynamics of the aligned molecules, and this reversal of $n(t)$ causes the re-emission of the signal.

medium. (ii) (absorption) By choosing an appropriate pump-signal delay, the weak signal pulse propagates in a region in time where the refractive index is approximately linear, i.e. $n(t) \approx n_{0}+\dot{n} t$. This evenly shifts the frequency distribution of the signal pulse by an amount at least equal to its bandwidth (for a sufficient $\dot{n}$ ), thereby scanning all its frequency components over the transition frequency of two-level atoms such that the entire pulse can be absorbed. (iii) (emission) Later in time, oscillations in the rotational dynamics of the molecules generate the opposite slope in the refractive index of the propagation medium, i.e. $n(t+\tau) \approx n_{0}-\dot{n} t$. This in turn causes constructive interference in the collective wave function of the two-level atoms, and results in re-emission of the signal with a delay $\tau$ that defines the storage time of the memory.

\section{B. Molecular alignment in the host gas: refractive index dynamics preparation}

A non-resonant ultrafast laser pump pulse excites a rotational wave packet $|\Psi(t)\rangle=\sum_{J, M} A_{J, M}(t)|J, M\rangle$ in a rigid-rotor type molecule, where $|J, M\rangle$ are orbital angular momentum eigenstates $\langle\theta, \phi \mid J, M\rangle=Y_{J M}(\theta, \phi)$, and $A_{J, M}(t)$ are the coefficients of the rotational excitations. The time evolution of the rotational wave packet is given by the Schrödinger equation [28]:

$$
i \frac{\partial}{\partial t}|\Psi(t)\rangle=\left[B_{0} \mathbf{J}^{2}-U_{0}(t) \cos ^{2} \theta\right]|\Psi(t)\rangle
$$

where $B_{0}$ is the rotational constant of the molecule, $\mathbf{J}^{2}$ is the rotational energy operator, $U_{0}(t) \cos ^{2} \theta$ describes the interaction between the (linearly polarized) electric field of the laser and polarizability of the molecule, and $\theta$ is the relative angle between the laser polarization vector and the molecular axis. Note that all equations are written in atomic units throughout the paper, unless otherwise specified. The magnitude of the laser interaction term is given by

$$
U_{0}(t)=\frac{1}{4} \Delta \alpha \mathcal{E}_{0}^{2} \sin ^{2}\left(\frac{\pi t}{2 \sigma_{p}}\right)
$$

where $\mathcal{E}_{0}$ is the pump field strength, $\sigma_{p}$ is the pump pulse duration, and $\Delta \alpha=\alpha_{\|}-\alpha_{\perp}$ is the difference in the polarizability of the molecule along its two axes.

The alignment generated by this rotational wave packet is characterized by the expectation value $\left\langle\cos ^{2} \theta\right\rangle(t)=\left\langle\psi(t)\left|\cos ^{2} \theta\right| \psi(t)\right\rangle$, and must be averaged over the thermal Boltzmann distribution of the molecules' initial angular momentum states at a temperature $T$. Specifically, each initial $|J, M\rangle$ state present in the initial thermal distribution is excited by the pump field and generates a particular $\left\langle\cos ^{2} \theta\right\rangle_{J, M}(t)$, where the added subscript denotes that this contribution came from the initial thermally-populated $|J, M\rangle$ state. These $\left\langle\cos ^{2} \theta\right\rangle_{J, M}(t)$ are then further averaged over the initial Boltzmann distribution to give the thermally-averaged $\left\langle\cos ^{2} \theta\right\rangle_{T}(t)$

$$
\left\langle\cos ^{2} \theta\right\rangle_{T}(t)=\frac{\sum_{J, M} g_{J} e^{-E_{J} / k_{B} T}\left\langle\cos ^{2} \theta\right\rangle_{J, M}(t)}{\sum_{J} g_{J}(2 J+1) e^{-E_{J} / k_{B} T}}
$$

where the rotational energies are $E_{J}=B_{0} J(J+1)$, and $g_{J}$ is a weighting parameter taking into account nuclear spin statistics. For an ensemble of molecules in thermal equilibrium, the value $\left\langle\cos ^{2} \theta\right\rangle_{T}=1 / 3$ corresponds to a randomly aligned ensemble whereas $\left\langle\cos ^{2} \theta\right\rangle_{T}=1$ $\left(\left\langle\cos ^{2} \theta\right\rangle_{T}=0\right)$ corresponds to an ensemble fully parallel (perpendicular) to the electric field of the pump.

\section{The Maxwell-Bloch equations}

We use a semi-classical treatment to propagate a weak signal pulse in a medium consisting of an ensemble of two-level atoms and a time-dependent molecular alignment. We define a signal pulse with a Gaussian temporal envelope and central frequency $\omega_{0}$ as

$$
E_{s}(t)=E_{0} e^{-4 \ln (2)\left(t-t_{0}\right)^{2} / \sigma_{s}^{2}} e^{-i \omega_{0} t}
$$

where $\sigma_{s}$ is the signal pulse duration at the full-widthhalf-max. The two-level atoms with energy spacing $\omega_{b a}$ and transition dipole matrix element $\mu_{b a}$ interact with the propagating signal. The resulting dynamics can be described via the Bloch equations [29]:

$$
\begin{aligned}
\dot{\rho}_{b a}(t) & =-\left(i w_{b a}+\frac{1}{T_{2}}\right) \rho_{b a}(t)-i \mu_{b a} E_{s}(t) \rho_{d}(t) \\
\dot{\rho}_{d}(t) & =\frac{-\rho_{d}(t)+1}{T_{1}}+2 i\left(\mu_{b a} E_{s}(t) \rho_{a b}-\mu_{a b} E_{s}(t) \rho_{b a}\right)
\end{aligned}
$$


where $\rho_{d}$ is the difference in the excited and ground state populations. The parameters $T_{1}$ and $T_{2}$ take into account the lifetime of the transition and dipole dephasing, respectively. Eq. 5] couples to Maxwell's wave equation (see below) via the total polarization of the medium $P(t)=P_{m}(t)+P_{a}(t)$, which includes effects due to the rotating molecules and the two-level atoms, respectively, i.e.

$$
\begin{aligned}
P_{m}(t) & =N_{m}\left[\alpha_{\perp}+\Delta \alpha\left\langle\cos ^{2} \theta\right\rangle_{T}(t)\right] E_{s}(t) \\
P_{a}(t) & =N_{a}\left[\mu_{a b} \rho_{b a}(t)\right]
\end{aligned}
$$

where $N_{m}$ and $N_{a}$ are the respective number densities. We assume a spatially-uniform distribution of both molecules and two-level atoms along the propagation axis.

The molecular polarization term generates a timedependent refractive index:

$$
n(t)=1+2 \pi N_{m}\left[\alpha_{\perp}+\Delta \alpha\left\langle\cos ^{2} \theta\right\rangle_{T}(t)\right] .
$$

which is valid for $N_{m} \ll 1$. Ignoring transverse dynamics, we use a simplified Maxwell equation

$$
\frac{\partial E(t)}{\partial z}+\frac{1}{c} \frac{\partial E(t)}{\partial t}=-\frac{2 \pi}{c} \frac{\partial P(t)}{\partial t}
$$

to solve for forward propagating (along $z$ ) waves [30, 31]. We note that this equation is applicable to the case of a single photon. Eq. 8 is solved in the moving reference frame of the pump pulse, i.e. $\tau \rightarrow t-\frac{z}{v_{s}}$ where

$$
v_{s}=\frac{c}{n_{0}}=\frac{c}{1+2 \pi N_{m}\left(\alpha_{\perp}+\Delta \alpha / 3\right)},
$$

such that the dynamics of the alignment always begin at $\tau=0$ in the reference frame of the pump. This assumes the pump is traveling through an ensemble of initially randomly aligned molecules with $\left\langle\cos ^{2} \theta\right\rangle_{T}=1 / 3$. Effects of the molecular alignment on the pump pulse itself do not strongly affect the generated rotational wave packet [32], hence we ignore any modifications to the pump pulse during propagation. In the current model, we neglect group velocity dispersion. For a specific implementation, care may need to be taken to manage its effects. We note, however, that in early studies of pulse compression using a similar setup, the effects of dispersion were not detrimental [19, 32, 33.

\section{NUMERICAL RESULTS AND DISCUSSION}

\section{A. Basic storage protocol}

Fig. 2 a shows the time-dependent refractive index generated by aligning an ensemble of $\mathrm{CO}_{2}$ gas molecules $\left(\alpha_{\perp}=1.97 \AA^{3}, \Delta \alpha=2.04 \AA^{3}\right.$ [34], $N_{m}=1 \times$ $\left.10^{21} \mathrm{~cm}^{-3}\right)$ at room temperature $(295 \mathrm{~K})$ with a strong $\left(5 \times 10^{13} \mathrm{~W} / \mathrm{cm}^{2}\right)$ non-resonant 50 fs pump pulse. Here we plot the refractive index as the deviation from unity

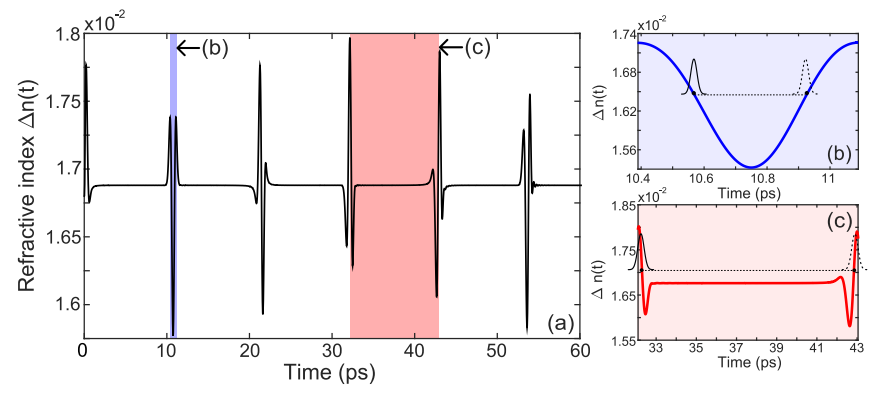

FIG. 2. (a) The alignment of an ensemble of $\mathrm{CO}_{2}$ molecules at room temperature using a strong non-resonant $50 \mathrm{fs}$ pump pulse generates a time-dependent refractive index $n(t)$ in the propagation medium. Here we plot $\Delta n(t) \equiv n(t)-1$. The molecules continue to rotate well after the pump pulse is off due to revival dynamics of the generated rotational wave packet. The highlighted regions (shown in (b) and (c)) provide a suitable refractive index modulation to achieve absorption and re-emission of a broadband photon. The memory in Fig. 3 is achieved using the refractive index in (b).

$\Delta n(t) \equiv n(t)-1$. The bandwidth of the pump should not overlap with the two-level atomic transition at $795 \mathrm{~nm}$, which could be readily achieved by using a wavelength in the 1 - $2 \mu \mathrm{m}$ regime. We note that our chosen density $N_{m}$ represents a pressure of $\sim 40$ bar which is achievable in a high-pressure gas cell. The oscillations seen in Fig. 20, which are called rotational revivals [17, 35], reflect the rotational alignment dynamics of the molecules: The peaks (dips) in $n(t)$ correspond to moments in time where the molecules are preferentially aligned parallel (perpendicular) to the polarization direction of the pump. By changing the delay between the pump and signal pulses, we select a region within $n(t)$ with the desired shape, as shown in Fig. 2b and Fig. 26. Although we report results for the refractive index in the former region only, any refractive index dynamic containing two opposite linear slopes separated in time, such as the latter region, is also suitable for the scheme.

Fig. 3a shows the E-field amplitude $|E(t)|$ of a weak 50 -fs pulse $\left(\omega_{0}=1.4 \mathrm{eV}\right)$ propagating in the reference frame of the pump. We set the field amplitude $E_{0}$ in Eq. 4 to be the amplitude of a single photon occupying a mode volume defined by interaction distance and the pulse spot size. However, we note that the specific quantitative value used for the magnitude of $E_{0}$ is not crucial as long as it is in the linear regime (i.e. negligible atomic excitation).

The linear time-dependent refractive index in the medium causes a spatially-dependent frequency shift (see Fig. 3b). The propagation distance required for the signal to be shifted by its entire bandwidth is determined by the slope of $n(t)$. For our parameters, we require a propagation distance of $\sim 1 \mathrm{~cm}$. Because the pulse propagates in a region where the refractive index is linear, the Gaussian shape of the frequency distribution is preserved as the pulse is frequency-shifted. As the signal 


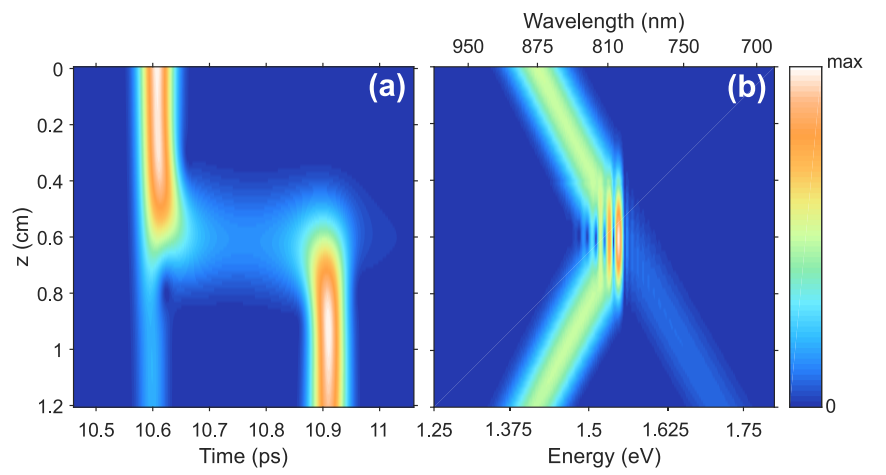

FIG. 3. Quantum memory for a weak 50 -fs pulse. In (a), the amplitude $|E(t)|$ of the signal is shown propagating in the reference frame of the pump in a medium containing two-level atoms and molecules generating the time-dependent refractive index $n(t)$ (shown in Fig. 20). This causes the signal to be frequency-shifted, as shown in (b): As the signal propagates, its frequency distribution is scanned over the transition wavelength $(795 \mathrm{~nm})$ of the two-level atoms such that the entire broadband pulse is absorbed. The linear slope of the refractive index eventually switches and the pulse is re-emitted from the medium, and again frequency-shifted, but in the opposite direction. Thus the dynamics of the refractive index cause the signal to be absorbed and then re-emitted from the medium with a delay $\tau$ that defines the storage time of the memory.

is scanned over the transition wavelength of the twolevel atoms $(795 \mathrm{~nm})$, it is completely absorbed for sufficiently high optical depths (see Fig. 6, here we use $N_{a}=1.35 \times 10^{16} \mathrm{~cm}^{-3}$ and $\left.\mu_{a b}=2.99 e a_{0}\right)$. We assume the two-level atoms have a negligible linewidth relative to the timescale of refractive index dynamics, and hence ignore relaxation processes $\left(T_{1}=T_{2} \rightarrow \infty\right.$ in Eq. 51) .

After some delay, which we here choose to label as $\tau$, the rotational dynamics of the molecules causes the refractive index gradient to switch (see Fig. 2b), and the signal is re-emitted from the medium, as shown in Fig. 3 a. Note that the opposite gradient in $n(t)$ also causes the emitted light to be frequency-shifted in the opposite direction, towards its initial distribution. The storage time is set by $\tau$, and in this simulation $\tau \approx 300 \mathrm{fs}$. The fringes in Fig. 3b are due to the fact that the absorbed and emitted pulses are two identical Gaussian functions separated by a time delay $\tau$, which in Fourier space transforms to a phase term $1+e^{i \tau \omega}$, and thus yields fringes whose spacing is given by the inverse of the storage time of the memory.

The timescale of the linear region of the refractive index oscillation in Fig. 2b sets the maximum length of the signal pulse, which in the case of $\mathrm{CO}_{2}$ is roughly $150 \mathrm{fs}$. We note that this is not a limitation of the scheme as molecular alignment can be achieved with many different molecules: oscillations in $n(t)$ depend on the moment of inertia of the molecule being aligned. For instance, a heavier molecule such as $\mathrm{Br}_{2}$ will rotate more slowly and hence the refractive index it generates will also have slower oscillations. One could also consider using adiabatic alignment [17] as an alternative to impulsive align- ment since the former operates with picosecond pump pulses.

Optical control of the molecular alignment process provides additional versatility to this scheme. For example, undesired dynamics in the alignment, such as the small oscillation at $32.5 \mathrm{ps}$ in Fig. 22 , can be minimized by temporal shaping of the pump pulse. Although we numerically verified that the slope in this feature is insufficient to cause re-emission of the pulse (not shown), it does affects the specific re-emission time at the subsequent revival at $\sim 43$ ps.

\section{B. Variable read times}

The memory scheme we have presented above effectively operates as a delay line with a fixed storage time. However, it can be extended to support variable storage times. It is possible to attenuate and regenerate the rotational revivals by using additional control pulses [25, 26]. Control over the alignment revival dynamics is shown in Fig. 4. Here, the initial alignment revivals generated by the pump pulse (seen in Fig. 4, same revivals as shown in Fig. 2(a)) are attenuated using a control pulse positioned at $21.45 \mathrm{ps}(\mathrm{b})-(\mathrm{d})$. Recall that $n(t)$ is proportional to $\left\langle\cos ^{2} \theta\right\rangle$ as shown in Eq. (7). The attenuating control pulse is identical to the initial pump pulse. This control pulse applies a torque to the molecules just as they are rotating toward the equatorial plane, and stops the rotations generated by the first pump pulse. The rotational distribution of angular momentum states is then effectively returned to the initial thermal distribution. The rotational revivals can now be regenerated at a later time. For example, in Figs. 4(c) and (d), the revivals are regenerated using a final control pulse (again identical to the first pump pulse) positioned at $40 \mathrm{ps}$ and $70 \mathrm{ps}$, respectively.

We now demonstrate controllable read times for the memory scheme using this control over the alignment revivals. Fig. 5 shows example calculations where the read time has been set to three different values. These calculations use a medium length of $4 \mathrm{~cm}$ and an atomic density of $N_{a}=1.6 \times 10^{16} \mathrm{~cm}^{-3}$. All remaining parameters are the same as used in Fig 3. Consider first Figs. [5](a)-(c). In these cases, the 50 fs signal pulse (red) arrives at 21.3 ps and is stored in the memory using the falling edge of concomitant revival. This revival is then immediately attenuated with a control pulse positioned at 21.45 ps. Panels (a)-(c) show cases where the stored signal is then read out at three different times. The read-out is triggered by regenerating the revivals at times 30,35 , and 40 ps respectively using a final control pulse. The reemitted signal pulses are shown in green. In the plots, the re-emitted pulses are seen to lag a bit (on the order of 0.1 to $0.2 \mathrm{ps}$ ) behind the read-out revival. This occurs because, once re-emitted, the signal pulse propagates in a region of $n(t)$ that has a higher value than the pump pulse, and hence the re-emitted signal starts to lag be- 

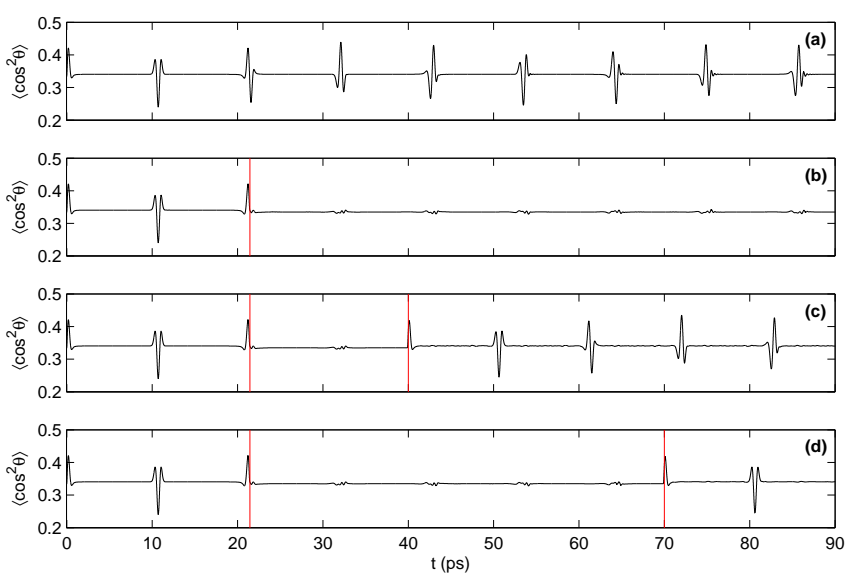

FIG. 4. Controlling the rotational revivals. The timings of the control pulses used in these examples are denoted by the vertical lines. (a) Initial alignment revivals generating by the first pump pulse (same revivals as seen in Fig. 2a). (b) Revivals are attenuated using a control pulse positioned at 21.3 ps . (c) Attenuated revivals are regenerated using a final control pulse at $40 \mathrm{ps}$. (d) Attenuated revivals are regenerated using a final control pulse at $70 \mathrm{ps}$.
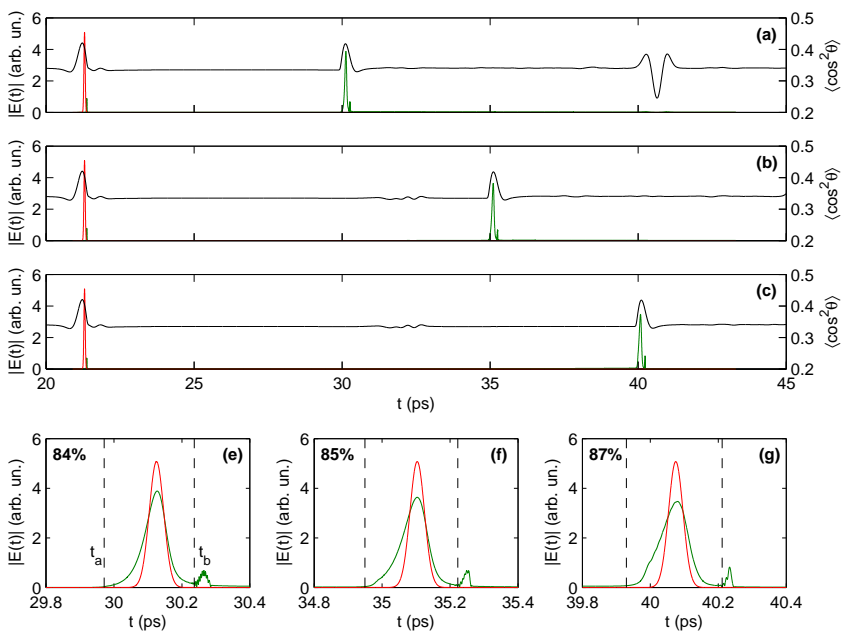

FIG. 5. Examples of variable read times. A signal pulse is stored in the memory at $21.3 \mathrm{ps}$ (red pulse). The memory is then read out at $30 \mathrm{ps}$ (a), $35 \mathrm{ps}$ (b), and $40 \mathrm{ps}$ (c). The re-emitted signal read out at these various times is shown in green. Panels (e-g) show close-ups of the emittied signal, along with a time-shifted copy of the intput signal for comparion. The percentages in panels (e-g) show the efficiencies of the quantum memory for each case.

hind the alignment revivals which are propagating with the speed of the pump.

Figures $5(\mathrm{e})-(\mathrm{g})$ show a closer view of the emitted signal pulses (green), together with a time-shifted copy of the initial signal pulse (red) for comparison. The reemitted signals are seen to be broader in time as compare to the initial signal. This is due to the fact that $n(t)$ is not perfectly linear; the non-linear components

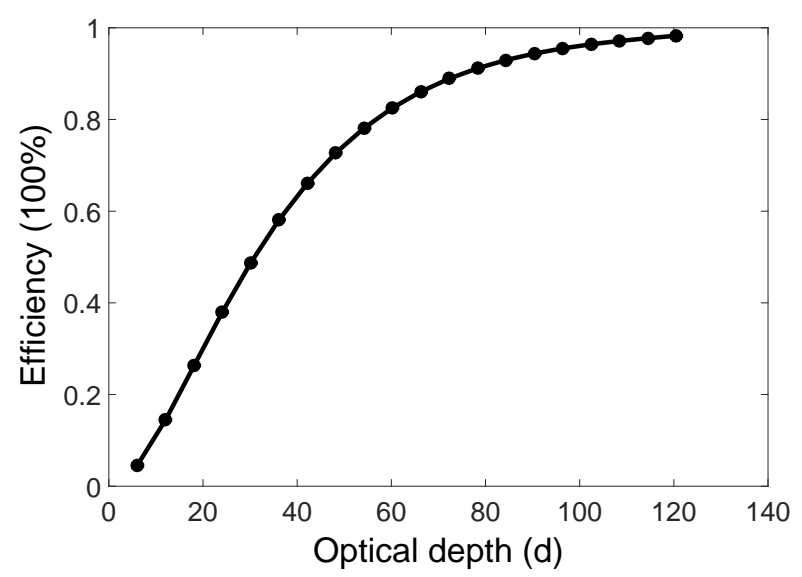

FIG. 6. Total memory efficiency as a function of optical depth of the medium, as defined in Eq. 11 High optical depths are required as the scheme involves storing large bandwidth pulses in an atomic transition with a narrow linewidth.

of $n(t)$ add dispersion to the propagation and effectively add chirp the signal. Also seen in Fig. 5(e)-(g) is a small post-pulse, which is a leakage process of the memory. For these cases, we also calculate the efficicency $\mathcal{E}$ of the memory by integrating over the intensities in the main part of the signal

$$
\mathcal{E}=\frac{\int_{t_{a}}^{t_{b}}\left|E_{\text {out }}(t)\right|^{2} d t}{\int\left|E_{\text {in }}(t)\right|^{2} d t}
$$

where $\left|E_{\text {out }}(t)\right|^{2}$ and $\left|E_{\text {int }}(t)\right|^{2}$ are the intensities of the output (emitted) and input (stored) signal pulses. The integration region over the main part of the output pulse, $t_{a}$ to $t_{b}$, is labeled explicitely in Fig. 5 (e), while in Figs $5(\mathrm{f})-(\mathrm{g})$ we denoted this region by the vertical dashed lines alone. The resulting efficiencies are shown as percentages in (e)-(g) and are between $84 \%$ to $87 \%$. Although not seen clearly, part of the deviation from $100 \%$ efficiency is due to less than perfect efficiency in the write step.

These examples show a quantum photonic memory that stores a 50 fs pulse for about $20 \mathrm{ps,} \mathrm{giving} \mathrm{a} \mathrm{time-}$ bandwidth product of approximately $20 \mathrm{ps} / 0.05 \mathrm{ps}=$ 400. The largest storage times acheivable is limited by the $T_{2}$ time of the two-level atoms, typically on the order of one nanosecond. For a 50 fs pulse, this leads to a time-bandwidth product of order $10^{4}$.

\section{Additional efficiency considerations}

The efficiency of the quantum memory is plotted as a function of optical depth in Fig. 6] The optical depth of the system is modified due to the effective inhomogeneous broadening of the medium. If we assume the refractive index varies linearly in the region where the pulse is absorbed and emitted, i.e. $n(t)=n_{0}+\dot{n} t$, then 
the optical depth is given by $[16]$

$$
d=\frac{2 \pi N_{a} \mu_{b a}^{2}}{\omega_{0}} \frac{n_{0}}{\dot{n}} \approx \frac{2 \pi N_{a} \mu_{b a}^{2}}{N_{m} \omega_{0} \Delta \alpha \frac{d}{d t}\left\langle\cos ^{2} \theta\right\rangle_{T}(t)}
$$

in the regime where $N_{m} \ll 1$. A substantial optical depth is required for high efficiency as the atomic ensemble is absorbing large-bandwidth pulses with a very narrow linewidth. Such optical depths have been achieved in hollow-core fibers containing warm atomic vapors [36, 37]. With this in mind, it is reasonable to use the $\mathrm{D}_{1}$ transition of an ensemble of ${ }^{87} \mathrm{Rb}$ atoms in a warm atomic vapor state for the gaseous atoms. The lifetime of this transition is on the order of nanoseconds and as such can be neglected in Eq. 5. Furthermore, we also note that room temperature diatomic molecules can be aligned in hollow-core fibers [38]. Compared to the above simulations that model a high-pressure cell regime, implementing the memory in a hollow-core fiber would force a lower molecular density $N_{m}$ and hence would require a longer propagation length on the order of $1 \mathrm{~m}$. Thus the proposed scheme could be achieved in either a highpressure gas cell a hollow-core fiber or containing a mix of a diatomic gas and a warm atomic vapor.

\section{CONCLUSION}

In summary, we explore the possibility of implementing a GEM-type quantum memory for ultrafast photons using molecular alignment to generate a time-dependent refractive index [16]: A linear refractive index is used to scan the frequency distribution of a broadband photon over the transition frequency of an ensemble of two-level atoms such that entire photon is absorbed. Later in time, the rotating molecules generate the opposite gradient in the refractive index and the photon is re-emitted. We numerically demonstrate that the proposed scheme can store a weak 50 -fs pulse for 400 times its duration in a gas mixture of ${ }^{87} \mathrm{Rb}$ (warm atomic) vapor and $\mathrm{CO}_{2}$ molecules at room temperature. This is achieved without the use of an external static field as required in previous GEM schemes. By exploiting the revival dynamics of the rotational wave packet generated in the alignment process along with additional control pulses, we envisage that the lifetime of such a memory can be extended to $T_{2}$ timescales of ${ }^{87} \mathrm{Rb}$, leading to time-bandwidth products of order $10^{4}$.

The proposed scheme explores one possible way of implementing an optical quantum memory in a medium with an ultrafast time-dependent refractive index. Inducing refractive index changes with ultrafast response times is also achievable via other mechanisms, such as the non-linear response of the electronic polarization of the medium [29] and in particular cross-phase modulation [39].

\section{ACKNOWLEDGMENTS}

The authors thank Euan Joly-Smith, Davor Curic, Rune Lausten, and, Paul Hockett for stimulating discussions.
[1] Khabat, H.; England, E.; Humphreys, P.; Bustard, P.J.; Acosta, V.; Nunn, J.; Sussman, B. J.; arXiv:1511.04018

[2] Bussières, F.; Sangouard, N.; Afzelius, M.; de Riedmatten, H.; Simon, C.; et al. J. Mod. Opt. 2013, 60 (18), 1519-1537.

[3] Sangouard, N.; Simon, C.; de Riedmatten, H.; et al. Rev. Mod. Phys. 2011, 83 (Mar), 33-80.

[4] Hammerer, K.; Sørensen, A.S.; Polzik, E.S. Rev. Mod. Phys. 2010, 82 (Apr), 1041-1093.

[5] England, D.G.; Bustard, P.J.; Nunn, J.; Lausten, R.; et al. Phys. Rev. Lett. 2013, 111 (Dec), 243601.

[6] England, D.G.; Fisher, K.A.G.; MacLean, J.P.W.; Bustard, P.J.; Lausten, R.; Resch, K.J.; et al. Phys. Rev. Lett. 2015, 114 (Feb), 053602.

[7] Bustard, P.J.; Lausten, R.; England, D.G.; et al. Phys. Rev. Lett. 2013, 111 (Aug), 083901.

[8] Reim, K.; Nunn, J.; Lorenz, V.; Sussman, B.; Lee, K.; Langford, N.; Jaksch, D.; et al. Nature Photon. 2010, 4 (4), 218-221.

[9] Saglamyurek, E.; Sinclair, N.; Jin, J.; Slater, J.A.; Oblak, D.; Bussieres, F.; George, M.; Ricken, R.; Sohler, W.; et al. Nature 2011, 469 (7331), 512-515.

[10] Hétet, G.; Longdell, J.; Alexander, A.; Lam, P.K.; et al. Phys. Rev. Lett. 2008, 100 (2), 023601.
[11] Moiseev, S.A.; Kröll, S. Phys. Rev. Lett. 2001, 87 (Oct), 173601.

[12] Kraus, B.; Tittel, W.; Gisin, N.; Nilsson, M.; Kröll, S.; et al. Phys. Rev. A 2006, 73 (2), 020302.

[13] Alexander, A.; Longdell, J.; Sellars, M.; et al. Phys. Rev. Lett. 2006, 96 (4), 043602.

[14] Hosseini, M.; Sparkes, B.M.; Hétet, G.; Longdell, J.J.; Lam, P.K.; et al. Nature 2009, 461 (7261), 241-245.

[15] Hedges, M.P.; Longdell, J.J.; Li, Y.; et al. Nature 2010, 465 (7301), 1052-1056.

[16] Clark, J.; Heshami, K.; Simon, C. Phys. Rev. A 2012, 86 (Jul), 013833.

[17] Stapelfeldt, H.; Seideman, T. Rev. Mod. Phys. 2003, 75 (Apr), 543-557.

[18] Sussman, B.J. Am. J. Phys. 2011, 79 (5), 477-484.

[19] Bartels, R.A.; Weinacht, T.C.; Wagner, N.; Baertschy, M.; Greene, C.H.; Murnane, M.M.; et al. Phys. Rev. Lett. 2001, 88 (Dec), 013903.

[20] Zhavoronkov, N.; Korn, G. Phys. Rev. Lett. 2002, 88 (May), 203901.

[21] Bustard, P.J.; Sussman, B.J.; Walmsley, I.A. Phys. Rev. Lett. 2010, 104 (May), 193902.

[22] Villeneuve, D.M.; Aseyev, S.A.; Dietrich, P.; Spanner, M.; Ivanov, M.Y.; et al. Phys. Rev. Lett. 2000, 85 (Jul), 542-545. 
[23] Wu, J.; Lu, P.; Liu, J.; Li, H.; Pan, H.; Zeng, H.; Appl. Phys. Lett. 2010, 97 (Oct), 161106.

[24] Shapiro, E.A.; Khavkine, I.; Spanner, M.; et al. Phys. Rev. A 2003, 67 (Jan), 013406.

[25] Spanner, M.; Shapiro, E.A.; Ivanov, M. Phys. Rev. Lett. 2004, 92 (Mar), 093001.

[26] Lee, K.F.; Villeneuve, D.M.; Corkum, P.B.; et al. Phys. Rev. Lett. 2004, 93 (Nov), 233601.

[27] Steck, D., Rubidium 87 D Line Data, http://steck.us/alkalidata 2008.

[28] Friedrich, B.; Herschbach, D. Phys. Rev. Lett. 1995, 74 (Jun), 4623-4626.

[29] Boyd, R.W. Nonlinear optics. Academic press: California, USA, 2003.

[30] Couairon, A.; Brambilla, E.; Corti, T.; Majus, D.; Ramírez-Góngora, O.d.J.; et al. Eur. Phys. J. Spec. Top. 2011, 199 (1), 5-76.

[31] Bullough, R.; Jack, P.; Kitchenside, P.; et al. Phys. Scr. 1979, $20(3-4), 364$.
[32] Spanner, M.; Ivanov, M.Y. Opt. Lett. 2003, 28 (7), 576578.

[33] Kalosha, V.; Spanner, M.; Herrmann, J.; et al. Phys. Rev. Lett. 2002, 88 (Feb), 103901.

[34] Miller, K.J. J. Am. Chem. Soc. 1990, 112 (23), 85438551.

[35] Seideman, T. Phys. Rev. Lett. 1999, 83 (Dec), 49714974.

[36] Kaczmarek, K.T.; Saunders, D.J.; Sprague, M.R.; Kolthammer, W.S.; Feizpour, A.; Ledingham, P.M.; Brecht, B.; Poem, E.; Walmsley, I.A.; Nunn, J.; Opt. Lett. 2015, 40 (Dec), 5582-5585

[37] Sprague, M.R.; England, D.G.; Abdolvand, A.; Nunn, J.; Jin, X.M.; Kolthammer, W.S.; Barbieri, M.; Rigal, B.; Michelberger, P.S.; Champion, T.F. et al. New. J. Phys. 2013, 15 (5), 055013.

[38] Sickmiller, B.A.; Jones, R.R. Phys. Rev. A 2009, 80 (Sep), 031802.

[39] Spanner, M.; Pshenichnikov, M.; Olvo, V.; et al. Appl. Phys. B 2003, 77 (2-3), 329-336. 